\title{
Development of the LunaH-Map miniature neutron spectrometer
}

Erik B. Johnson, Craig Hardgrove, Richard Starr, Sam Vogel, Rebecca Frank, et al.

Erik B. Johnson, Craig Hardgrove, Richard Starr, Sam Vogel, Rebecca Frank, Graham Stoddard, Stephen West, James Christian, "Development of the LunaH-Map miniature neutron spectrometer," Proc. SPIE 10392, Hard XRay, Gamma-Ray, and Neutron Detector Physics XIX, 103920H (24 August 2017); doi: 10.1117/12.2276273

Event: SPIE Optical Engineering + Applications, 2017, San Diego, California, United States 


\title{
Development of the LunaH-Map Miniature Neutron Spectrometer
}

\author{
Erik B. Johnson ${ }^{* a}$, Craig Hardgrove ${ }^{\mathrm{b}}$, Richard Starr ${ }^{\mathrm{c}}$, Sam Vogel ${ }^{\mathrm{a}}$, Rebecca Frank ${ }^{\mathrm{a}}$, Graham \\ Stoddard ${ }^{\mathrm{a}}$, Stephen West ${ }^{\mathrm{b}}$, and James Christian ${ }^{\mathrm{a}}$ \\ ${ }^{a}$ Radiation Monitoring Devices, 44 Hunt Street, Watertown MA 02472; ${ }^{b}$ University of Arizona, \\ School of Earth and Space Exploration, Tempe, AZ 85287-6004, ${ }^{\mathrm{c} C}$ Catholic University of America, \\ 620 Michigan Ave., N.E. Washington, DC 20064
}

\begin{abstract}
There is strong evidence that water-ice is relatively abundant within permanently shadowed lunar surface materials, particularly at the poles. Evidence for water-ice has been observed within the impact plume of the LCROSS mission and is supported by data gathered from the Lunar Exploration Neutron Detector (LEND) and the Lunar Prospector Neutron Spectrometer (LPNS). Albedo neutrons from the Moon are used for detection of hydrogen, where the epi-thermal neutron flux decreases as hydrogen content increases. The origin on the concentration of water within permanently shadowed regions is not completely understood, and the Lunar Polar Hydrogen Mapper (LunaH-Map) mission is designed to provide a high-resolution spatial distribution of the hydrogen content over the southern pole using a highly elliptical, low perilune orbit. The LunaH-Map spacecraft is a $6 \mathrm{U}$ cubesat consisting of the Miniature Neutron Spectrometer (Mini-NS). Mini-NS is not collimated, requiring a low altitude to achieve a higher spatial resolution compared to previous missions. To develop a compact neutron detector for epi-thermal neutrons, the Mini-NS comprises of 2-cm thick slabs of CLYC $\left(\mathrm{Cs}_{2} \mathrm{LiYCl}_{6}\right)$, which provide a sensitivity similar to a 10-atm, $5.7-\mathrm{cm}$ diameter He-3 tubes, as used in LPNS. The Mini-NS digital processing electronics can discriminate by shape and height to determine signal (albedo neutrons) from background (cosmic rays). The Mini-NS achieves a total active sensing area of $200 \mathrm{~cm}^{2}$ and is covered with a cadmium sheet to shield against thermal neutrons. The research and development on the detector modules show a robust design ready for space flight.
\end{abstract}

Keywords: lunar hydrogen, neutron spectrometer, moon, CLYC, water-ice

\section{INTRODUCTION}

There are clear observations of water ice on the lunar surface [1-3]. The Lunar CRater and Observation Sensing Satellite (LCROSS) observed larger concentrations of water after impacts from the Centaur booster and Shepherding Spacecraft at the lunar southern pole $[4,5]$. Data from the Lunar Reconnaissance Orbiter's (LRO) Lunar Epithermal Neutron Detector (LEND) and Lunar Prospector Neutron Spectrometer (LPNS) indicate a heterogeneous distribution of water on scales less than $10 \mathrm{~km}$ [6-10]. A primary tool for mapping the water concentration within lunar soil is neutron detection, where the neutrons are generated from nuclear scattering of galactic cosmic rays (primarily protons) within the soil. The neutron spectrum generated will be moderated by the soil, and as the hydrogen content increases, the moderation will soften (or thermalize) the spectrum to a greater extent. Counting thermal and epithermal neutrons, spatial and depth information on the hydrogen (water) content is possible, while counting the epi-thermal neutrons alone will provide spatial information. With a known distance of a neutron detector from the surface, geometrical constraints provide a map of the neutrons emitted from the surface as a spacecraft orbits. A side from prospecting, the process leading to the water distribution on the lunar surface, particularly in permanently shadowed regions, is not fully understood. A spatial map with high spatial resolution beyond existing data sets of the southern pole (same pole as the LCROSS impact) will provide data to understand these processes. This is obtained by flying a neutron detector close (down to $5 \mathrm{~km}$ ) to the lunar surface.

*ejohnson@rmdinc.com; phone 1-617-668-6886; rmdinc.com

Hard X-Ray, Gamma-Ray, and Neutron Detector Physics XIX, edited by Arnold Burger, Ralph B. James,

Michael Fiederle, Larry Franks, Stephen A. Payne, Proc. of SPIE Vol. 10392, 103920H

(c) 2017 SPIE · CCC code: 0277-786X/17/\$18 - doi: 10.1117/12.2276273 


\section{DESIGN OVERVIEW}

A compact $6 \mathrm{U}$ CubeSat consisting of a compact neutron detector called the Miniature Neutron Spectrometer (MiniNS) has been designed for the Lunar Polar Hydrogen Mapper (LunaH-Map) mission to achieve these studies. A drawing of the LunaH-Map CubeSat is in Figure 1a). Stripping away the cubesat parts, the instrument is shown in Figure 1b) to show the mechanical structure of the Mini-NS. The instrument consists of two detectors that comprise of four modules (or sensors), which is better observed in Figure 1c). The modules (Figure 1d) consist of a hermetically sealed CLYC $\left(\mathrm{Cs}_{2} \mathrm{LiYCl}_{6}\right)$ scintillator in an aluminum can, and the design provides $200 \mathrm{~cm}^{2}$ of the scintillator's area facing the Moon. The geometry allows space for propulsion and the readout electronics.
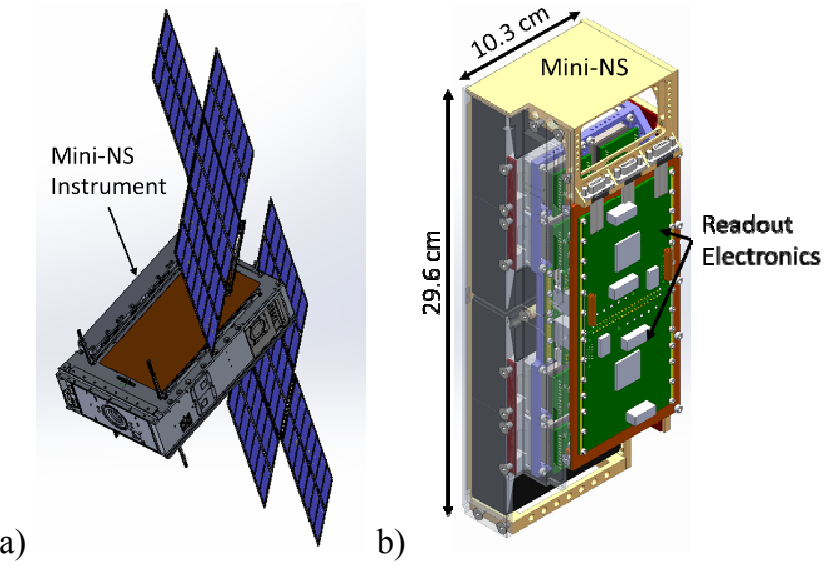

b)

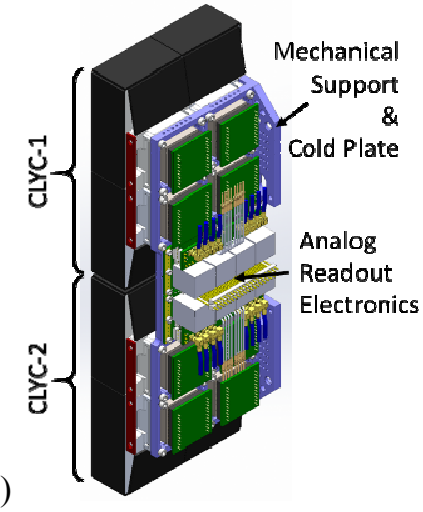

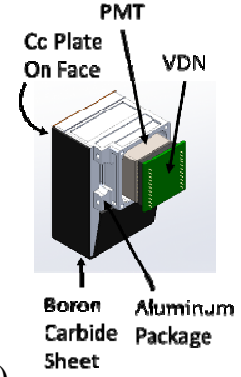

d)

Figure 1. a) CAD of the 6U LunaH-Map CubeSat with the solar arrays fully deployed. The neutron spectrometer (Mini-NS) location is pointed out in the design. b) The mechanical structure of the Mini-NS. The near-side panel is drawn as transparent to see into the instrument. c) The outer mechanics are removed showing the eight detector modules and inner readout electronics. d) The detector module is a packaged CLYC scintillator with a compact, square PMT attached to one corner.

A typical neutron detector for these missions is a He-3 tube, and the LPNS He-3 tubes compressed to 10 atm are a good reference for comparison. The tubes have flight heritage, and one tube can reside within the allocated volume of the neutron spectrometer. The effective area of the LPNS tube is roughly $114 \mathrm{~cm}^{2}$ for a mono-directional flux of neutrons from the lunar surface. The total area of the CLYC scintillators is $200 \mathrm{~cm}^{2}$, and for a quick comparison, the LPNS tube (He-3 at $10 \mathrm{~atm}$ ) shows a low area-efficiency product. If the area of the He-tube were increased to $200 \mathrm{~cm}^{2}$, CLYC with a thickness of $2 \mathrm{~cm}$ would effectively be equivalent (see Figure 2a). Dry soil compared to wet soil will affect the neutron spectrum differently, where wet soil will result in a smaller overall signal above $0.4 \mathrm{eV}$ and a larger signal below that energy compared to dry soil. Cadmium (Cd-113) has a high cross section below $0.2 \mathrm{eV}(>10,000 \mathrm{~b})$ and drops to below $10 \mathrm{~b}$ above $10 \mathrm{eV}$ and is an ideal candidate to isolate the signal of interest (the energy integrate neutron counts above $0.4 \mathrm{eV})$.

He-3 is inherently inefficient for detection of gamma rays but will generate a signal for protons, albeit the flux is low for GCR protons. CLYC is a dual, gamma-ray/neutron detector, making it very sensitive to both gamma rays and charged ions. Simulations show that the background from GCR is dominate above $8 \mathrm{MeV}$ with an expected detection rate two orders below the signal detection rate. Figure $2 \mathrm{~b}$ shows the discrimination capability of CLYC, as the neutrons are well isolated from the gamma rays. The discrimination is done using a pulse shape analysis method. The majority of protons will populate the plot in the gamma ray band above $6 \mathrm{MeV}$. Background sources may include neutrons generated in the spacecraft from GCR, lunar neutrons scattered off of the spacecraft into the detector, and low energy protons that deposit energies equivalent to the neutron signal. 

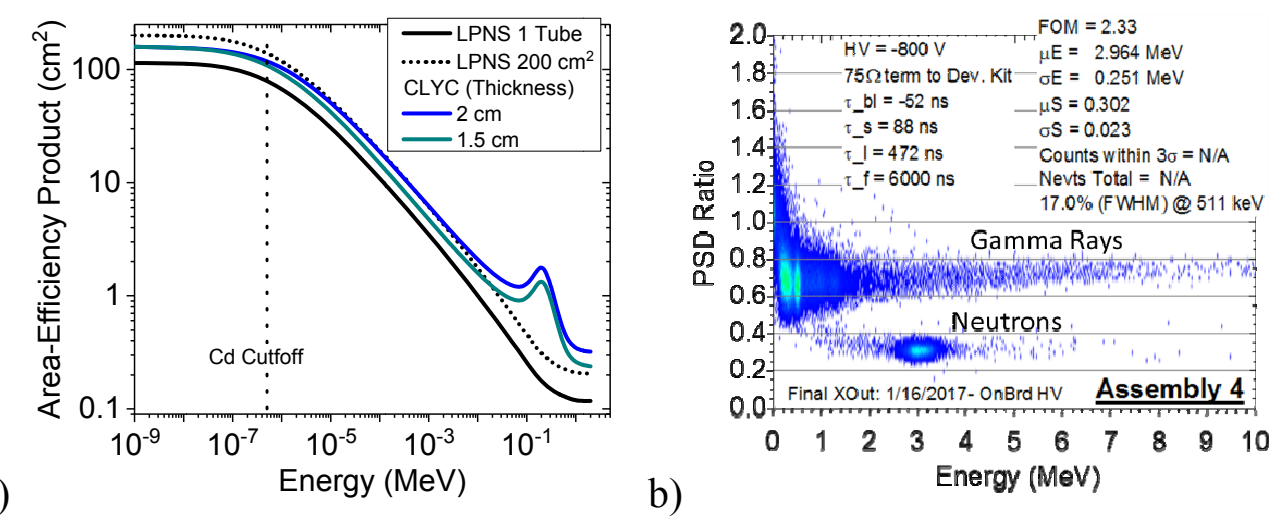

Figure 2. a) Area-efficiency of the CLYC detectors compared to a He-3 tube from LPNS. The black lines are from the LPNS, with the dashed line scaled to $200 \mathrm{~cm}^{2}$. The other lines are for a $200 \mathrm{~cm}^{2}$ of CLYC with a thickness of $2 \mathrm{~cm}$ and 1.5 $\mathrm{cm}$. b) Plot of the pulse shape discrimination (PSD) ratio verses the energy deposited for electrons (gamma rays). Due to light quenching in the scintillator, the energy deposited for the neutrons $(\sim 4.8 \mathrm{MeV})$ is higher than indicated in the plot.

The CLYC modules are coupled to compact, Hamamatsu R11265 photomultiplier tubes (PMT). The signals from the detectors are connected to analog electronics that set the voltage for the PMTs and amplifiers for processing the signal. The signals from the analog electronics are sent to a digital board, where these boards are separated to minimize heat loading on the CLYC. The digital boards digitize the signals, capture and process the signals with a FPGA, store data, and interface with the spacecraft. The instrument has two thermal electrical coolers (TEC) to reduce the rate of change in temperature during data acquisition. The operation is designed to bleed current into the TECs such that switching noise from a modulated signal in the controller is not present during data acquisition. The mass of the instrument is 3.3 $\mathrm{kg}$ with a power budget estimated at $22 \mathrm{~W}$ when both of the TECs are conducting $1 \mathrm{~A}$ each.

The initial design of the instrument focused on the design of the module. The selection of the PMTs was dependent on their ability to survive the launch, and the crystals inside the packaging need to survive the launch as well as temperature fluctuations.

\section{COMPONENT DEVELOPMENT}

\subsection{Photomultiplier Tubes}

The geometry of the detector modules was reviewed, and as the primary goal of the mission is to measure neutrons. Gamma-ray spectroscopy is not required. For a good gamma-ray spectrometer, light collection from the scintillator is critical, and the ideal condition is to place a smaller crystal on a larger PMT to capture as much light as possible. In our design, we know that the light yield from a neutron is large, and we can sacrifice light collection efficiency. We are limited in space, yet we want to fill as much of the volume with CLYC. From Figure 2, the thickness of the CLYC should be $2 \mathrm{~cm}$, and simulations have shown that the neutron detection efficiency has diminishing returns above $2 \mathrm{~cm}$, as the mass increases linearly. Hamamatsu recently developed a new PMT (R11265) similar to the R7600, but this tube has a larger active area and a smaller height. A primary cause of failure for PMTs is that the vibrations during launch will result in deformation of the dynode structures in the tube. The R11265 tubes were sent to AMES for vibration testing. The picture in Figure 3 shows the PMT mounted on the vibration table, and the tube was exposed to a sine wave before and after exposure to GEVS spectra on all three axis. The PMT was mounted with thermal gap pads ( $1 \mathrm{~mm}$ thick prior to compression) to suppress some of the vibration shock on the tube. The accelerometers mounted on the tube are used to identify any changes in the sine wave vibrational tests, to which no significant changes where observed.

Additional radiometric testing was conducted on the function of the PMT. A control CLYC crystal was used to measure the uncalibrated spectrum from a ${ }^{22} \mathrm{Na}$ source. The plot in Figure 3 shows the channel number captured by a multichannel analyzer (MCA) setup. The setup used a charged sensitive amplifier and a shaping amplifier, as with a typical nuclear detector readout. The location of the 511 and $1275 \mathrm{keV}$ peaks were captured before and after the vibration testing. The stable results give us high confidence that these PMTs will be successful on this mission. 


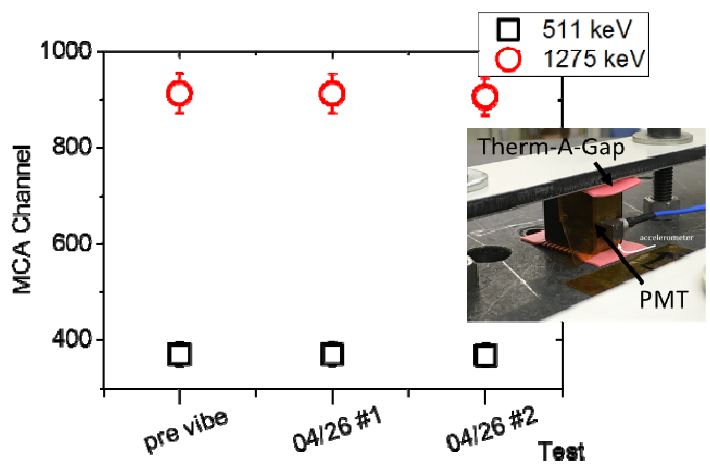

Figure 3. The inset picture (taken by Dr. Cook at AMES) shows the PMT mounted on the vibration table. The plot shows the MCA full energy peak locations in channel number (uncalibrated) as a function of pre- and post-vibe. The error bars are the fit errors to the full energy peaks.

The instrument has a $32 \mathrm{~W}$ requirement on the spacecraft, and the design process has indicated that the power draw is estimated to not to exceed $22 \mathrm{~W}$. Even though there is power available, the major concern for the small spacecraft is temperature. Operation of the electronics will result in the instrument to rapidly increase in temperature. From Figure 1c), there is a part that is labelled as a cold plate. This is connected to a thermal electric cooler to minimize the heat loading into the analog electronics and crystals. The hotter digital electronics are thermally isolated from the analog components. To further reduce the power on the system, the PMT's voltage divider networks (VDN) were tweaked from the Hamamatsu standard product. The tweak increased the total resistance of the network, and in turn, the volume of the custom part was reduced compared to the Hamamatsu product, as the part is not potted in the same way. Increasing the resistance on a VDN, the linear response of the PMT can be affected. The VDN is used to provide a voltage across the PMT dynode stages, where a constant current draw from the bias voltage (-800V) to ground results in a predictable voltage at each dynode stage. At high event rates, the current generated at each stage can lead to additional voltage drops across the network, which can lead to either an increase or decrease in gain depending on all of the terms associated with the circuit. The expected rate on each detector module (Figure 1d) will be less than $10 \mathrm{kcps}$ (estimated rate is closer to $1 \mathrm{kcps})$.

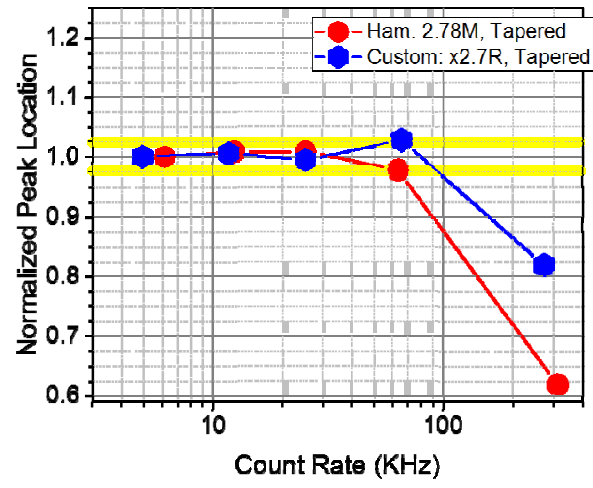

Figure 4. The plot of the normalized peak as a function of average event rate. The red point are from the standard Hamamatsu VDN product, and the blue hexagons are from the custom VDN.

A prototype circuit was fabricated and connected to the R11265 photomultiplier tube. A 1-cm cube of CLYC was optically attached to the PMT, and the event rate was measured on an oscilloscope when the detector was exposed to a ${ }^{22} \mathrm{Na}$ source. The distance was measured, and we assume that the source is point-like. The centroid of the $511 \mathrm{keV}$ peak was measured as the source was moved in line with the detector. The rate was referenced to the distance, and a correction was applied at the higher event rates where the oscilloscope reported a lower rate than expected. The custom VDN (blue hexagons) tested increased the resistance by a factor of 2.7. The results in Figure 4 show that the detector will provide a linear response for rates exceeding $10 \mathrm{kcps}$. The data is normalized to one from the lowest rate data point for each data set separately. An important note is that the rate identified here is an average, which is critical that the system is linear beyond $10 \mathrm{kcps}$. 


\subsection{CLYC Response to Ionizing Radiation}

The method used to detect neutons with this system will rely on CLYC. The primary reaction for detection of neutrons is the ${ }^{6} \mathrm{Li}(\mathrm{n}, \alpha) \mathrm{t}$ reaction which has a $\mathrm{Q}$ value of $4.8 \mathrm{MeV}$. The alpha and triton generate a light flash in the CLYC crystal that is unique compared to gamma pulses. The detection efficiency for this process in CLYC maximizes at $80 \%$ for thermal neutrons, as other processes in CLYC will stop the neutrons. One of the other processes in CLYC that will produce a neutron like signature is ${ }^{35} \mathrm{Cl}(\mathrm{n}, \mathrm{p})^{35} \mathrm{~S}$ with a $\mathrm{Q}$ value of $0.6 \mathrm{MeV}$. The protons from this process are very similar to the alpha and triton particles, which is indicated in Figure 5. The pulse shapes from the CLYC crystal was processed differently compared to Figure 2 , which is why the neutrons are above the gamma rays. The fact that the crystal is sensitive to both neutrons and gammas, suggests that the gammas will create false positives of neutron counts. The separation of the gamma and neutron distributions is shown in Figure 5. This separation is quantified using a figure of merit (FOM), which is the difference of the means of the distribution over the sum of the full width half maximum. From [11], a discussion on the relation of the FOM to the rejection of gammas is presented. From Figure 5, if a cut in the full integral is made and a histogram of the PSD ratio is generate after the cut is apply, two Gaussian-like distributions would be generated. One distributions is associated with neutrons, and the other is for gammas. It is calculated that when the number of gamma rays are a factor of $10^{6}$ larger than the number of neutrons, a FOM will provide a rejection ratio of $10^{-12}$. This rejection ratio is the number of gamma rays expected for each measured neutron. In other words, $10^{12}$ neutrons would need to be counted before the probability of detecting one gamma ray as a false positive approaches $100 \%$. For this project, low energy protons $(<5 \mathrm{MeV})$ can produce a signal similar to neutrons, which is part of the background contribution to the instrument.

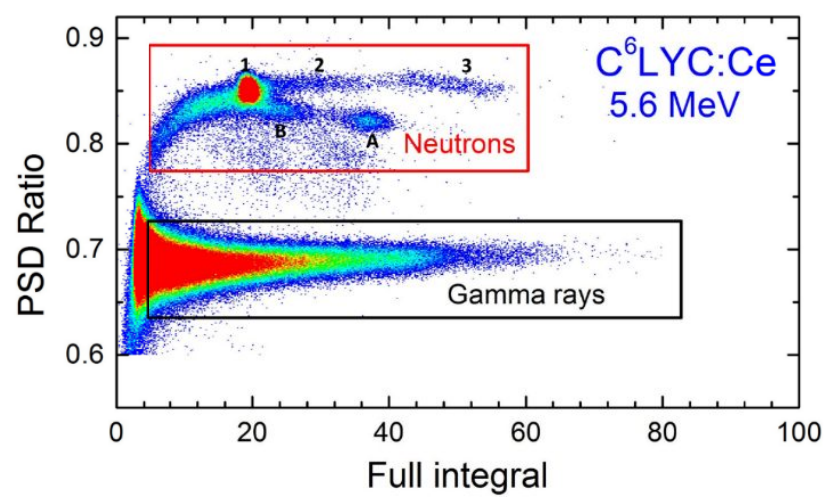

Figure 5: The pulse shape discrimination (PSD) ratio, which is a ratio of the leading component to the tail component of the pulse, versus the full integral (energy deposited). The neutron and gamma regions are indicated separately, as the detector was exposed to mono-energetic 5.6 MeV neutrons. The neutron distribution is complicated through various reactions. Reaction 1 (thermalized neutrons, stopped alphas and triton): ${ }^{6} \mathrm{Li}(\mathrm{n}, \alpha)^{3} \mathrm{H}, \mathrm{Q}=4783 \mathrm{keV}$. Reaction 2 (energetic neutrons, stopped alphas): ${ }^{35} \mathrm{Cl}(\mathrm{n}, \alpha)^{32} \mathrm{P}, \mathrm{Q}=938 \mathrm{keV}$. Reaction 3 (energetic neutrons, stopped alpha and triton): ${ }^{6} \mathrm{Li}(\mathrm{n}, \alpha)^{3} \mathrm{H}, \mathrm{Q}=4783$ $\mathrm{keV}$. Reaction A (energetic neutrons, stopped protons) ${ }^{35} \mathrm{Cl}(\mathrm{n}, \mathrm{p}){ }^{35} \mathrm{~S}, \mathrm{Q}=615 \mathrm{keV}$. Reaction $\mathrm{B}$ (energetic neutrons, stopped protons, population of ${ }^{35} \mathrm{~S}$ first excited state, $\left.1572 \mathrm{keV}\right):{ }^{35} \mathrm{Cl}(\mathrm{n}, \mathrm{p})^{35} \mathrm{~S}^{*}, \mathrm{Q}=-957 \mathrm{keV}$. The indicated neutrons below the reaction 1 peak in terms of the full integral are associated with energetic neutrons, whether the neutron was moderated or the charged particle did not stop in the crystal.

A beam test was conducted on CLYC crystals at Massachusetts General Hospital (MGH) proton therapy beam, where the beam was adjusted for two different energies. The accelerator operates nominally close to $200 \mathrm{MeV}$, where moderators are used to reduce the energy. A beam at $187 \mathrm{MeV}$ and a beam of $52 \mathrm{MeV}$ protons was incident on the detector. The data shows that CLYC will produce a gamma-ray like response for high energy (low linear energy transfer) protons, but as the energy is reduced to $52 \mathrm{MeV}$, the response shows the point where the shape starts to look more like a neutron. For comparison, the detector captured gamma-rays and neutrons from an AmBe source.

The analysis of the data Figure 6 show a linear increase in the PSD ratio as a function of energy. This is due to the methods and analysis methods and not a product of the crystal response to the protons. The feature may be due to saturation of the ADC or integration times that are not optimized.

The PSD will isolate the neutrons from gamma rays very well, but as we see in Figure 5 and Figure 6, low energy protons can produce neutron like responses. The beam tests are done at normal incidence, yet at shallow angles, the 
energy deposition will be lower. This can be imagined by translating the $52 \mathrm{MeV}$ proton response down to the neutrons in Figure 6. There are two factors that will help reduce this background. The first factor is that the crystal is a flat shape where the very few particles will traverse less than $2 \mathrm{~cm}$ through the material. The environment will not consist of a large number of these protons, and simulations show that the galactic cosmic ray (GCR) contribution at the neutron energy regime is two orders of magnitude less than the signal, and this is without pulse shape discrimination.
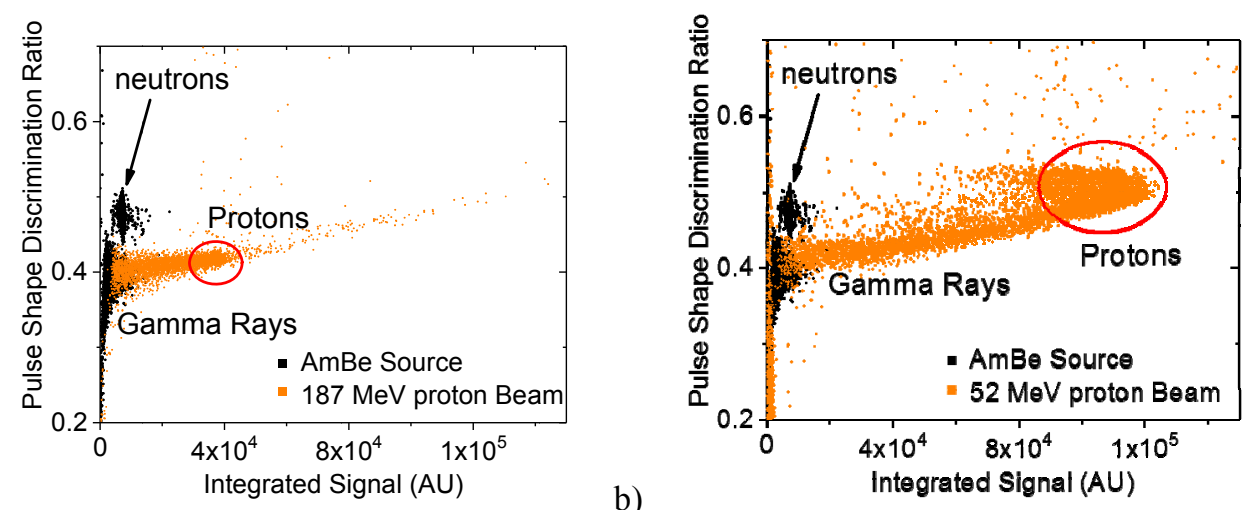

Figure 6: a) The pulse shape discrimination ratio as a function of energy deposited in arbitrary units (AU) for $187 \mathrm{MeV}$ protons and AmBe gamma rays and neutrons. b) The PSD ratio as a function of energy deposited for the $52 \mathrm{MeV}$ proton beam plotted with the same AmBe data in a).

A second beam at MGH was used to test the radiation tolerance of the CLYC. During the test, SrI was placed in the beam as well. The beam was set to $200 \mathrm{MeV}$ and dose on the CLYC were $8 \mathrm{kRad}, 80 \mathrm{kRad}$, and $800 \mathrm{kRad}$. Each of the crystals were used to measure the full energy peaks from a ${ }^{22} \mathrm{Na}$ source. Figure 7 shows the crystals that were exposed in the proton beam. The CLYC crystals are getting progressively darker, as the SrI turns yellow at a dose of $800 \mathrm{kRad}$. For comparison, expected dose on one of the LunaH modules is expected to be less than $100 \mathrm{Rad}$ from trapped radiation and GCR. A solar event can result in doses up to $250 \mathrm{Rad}$, and for the short mission duration ( $\sim 570$ days $)$, the planned dose is less than $1 \mathrm{kRad}$. With a light loss of $40 \%$ from $8 \mathrm{kRad}$ dose, CLYC will dim over this period by no more than $95 \%$ of the original light yield.

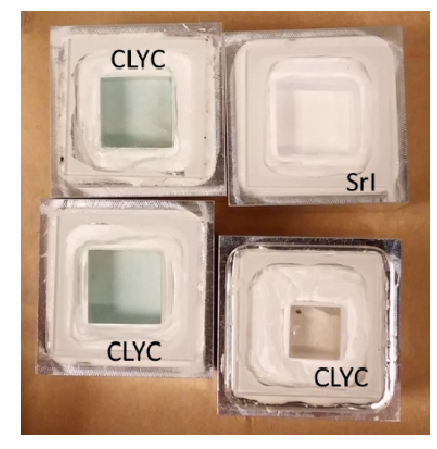

$8 \mathrm{kRad}$

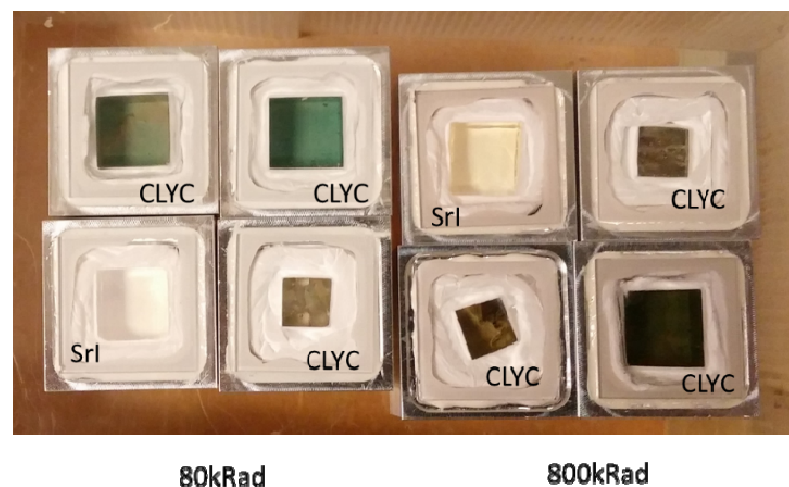

80kRad
$800 \mathrm{kRad}$

Figure 7: The pictures show the crystals right after exposure to $200 \mathrm{MeV}$ protons for three different doses.

For future missions, there is potential that CYLC may be annealed. The data presented here is from one crystal that was exposed to $8 \mathrm{kRad}$ of dose. The full energy peaks from ${ }^{22} \mathrm{Na}$ exposure were measured after the crystal was heated to 60 ${ }^{\circ} \mathrm{C}$ for a variety of times. Between the annealing, the crystals were placed within a port on an integrating sphere. A laser driven light source produces the light, which is sent into a monochormator. From there, the light is piped to the integrating sphere via a multimode fiber, where a small aperture is used to direct the light into the center of the mounted crystal. The light generated in the sphere is captured by a photodiode at $90^{\circ}$ to the fiber input and the crystal. This provides a measure of the light absorption/reflection through the crystal and off the Teflon back surface. The result shows that the CLYC crystal has measurable gains as a function of annealing time at $60{ }^{\circ} \mathrm{C}$ (see Figure 8 ). There is a linear correlation to the relative light yield measured using the ${ }^{22} \mathrm{Na}$ source. 

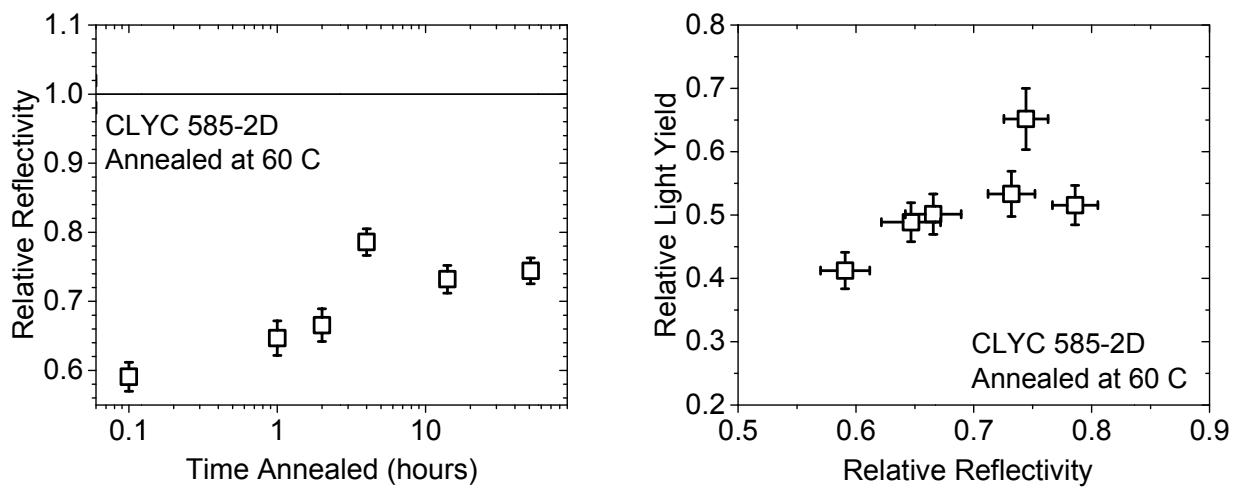

Figure 8: The left plot is the relative reflectivity at $500 \mathrm{~nm}$ as a function of annealing time for the CLYC 585-2D crystal, and the right plot is the relationship between the reflectivity measurement and the relative light yield.

\subsection{Initial Vibration Testing with CLYC}

An early prototype of the detector module (Figure 9a) was fabricated and sent AMES for vibrational testing. The modules were exposed to vibration from sine waves and GEVS standard random vibe on all three axis. The module wias connected to a data acquisition system to capture pulses and process the pulse shape discrimination (PSD) ratio and energy deposited. The background was used to assess the health of the detector. We observe a few neutrons in the spectrum, as shown in Figure 9b prior to vibration. The spectrum was collected after each test, and the data in Figure 9c) show no significant changes in the performance of the detector. As the health of the module was not at the same level as indicated in Figure 2, the testing provides a level of moderate confidence.
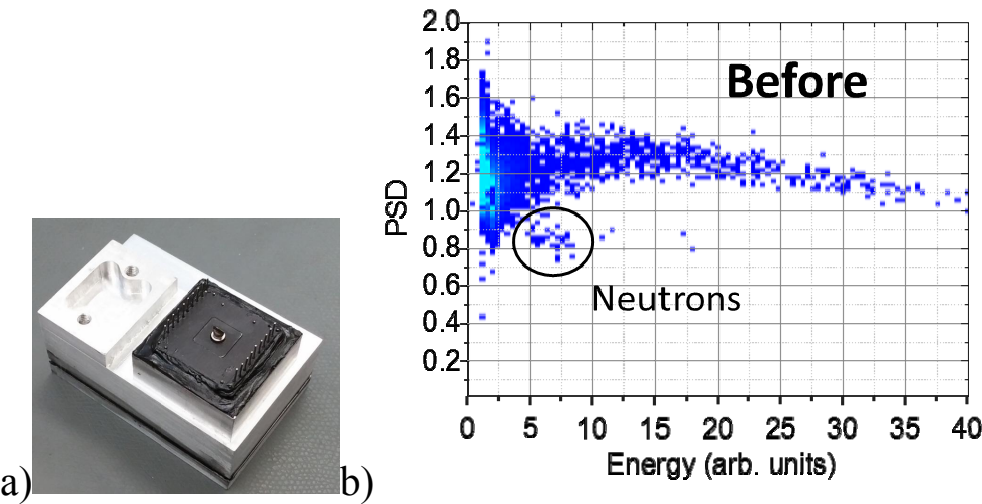

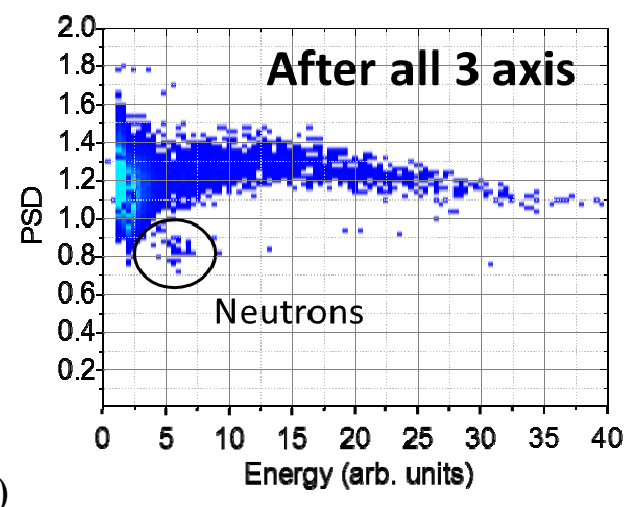

c)

Figure 9: a) picture of the module sent to AMES for vibration testing. b) Plot of the PSD ratio versus energy deposited of background events at AMES prior to vibration. c) Plot of the PSD ratio versus energy deposited of background after all testing.

\subsection{CLYC Thermal Considerations}

The detector will be exposed to temperatures ranging from $-30{ }^{\circ} \mathrm{C}$ to $50{ }^{\circ} \mathrm{C}$, and as part of the design, it is required that the detector remain at a reasonable stable temperature. The initial concern is that the response of the crystals will change as a function of temperature. This is observed in Figure 10. The figure of merit (FOM) changes as a function of temperature, which is due to changes in the pulse shape. Like other scintillation materials, the light yield also changes. If the temperature is varied by more than $10{ }^{\circ} \mathrm{C}$, additional design considerations are required to adjust the system parameters to locate and count neutrons. The neutron peak seen in the previous plot would be moving around on both axis too much. The system inherently accounts for this by capturing and storing the event by event data as well as the temperature, but in the scenario that the full data set cannot be transferred back to Earth, a much smaller (1000x) data set is stored. This smaller dataset is processed counts of background and neutron information. To keep the systematic errors low, the temperature should be kept stable to within $10{ }^{\circ} \mathrm{C}$. With the short data acquisition time ( $\left.\sim 30 \mathrm{~min}\right)$ per orbit, the temperature change has been modelled to be less than $10^{\circ} \mathrm{C}$. 

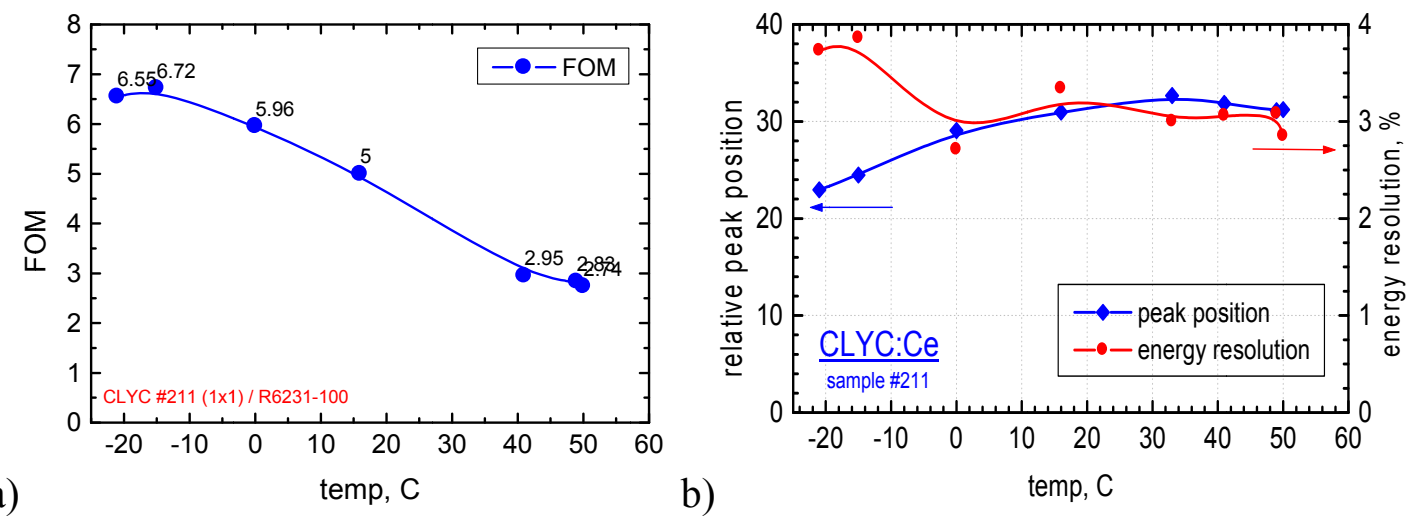

Figure 10. a) the Figure of merit as a function temperature. temperature.

b) the light yield and energy resolution as a function

The early development of the modules did expose an issue in handling CLYC. The material has grain boundaries along the growth axis. When the crystal is mechanically stress, fractures will occur along this axis. This is advantageous for the material in fabricating robust detectors. Though the crystal may crack, the all of the light generated will make it to the photodetector, when the photodetector is place on face perpendicular to this growth axis. As we are off to one corner, a crystal that has cracked will not provide a light channel out to the PMT. The result looks like the crystal forms different regions with divergent light yields. This is observed in Figure 11, where multiple neutron peaks are present. Though the neutron peaks are still observed, the larger region of interest for tallying neutron counts will result in a worse signal to noise. A picture of the damage to the crystal is shown in Figure 12, where prior to thermal cycling the module has two cracks below the PMT window. After the cycling the number of cracks significantly increase (see Figure 12b).

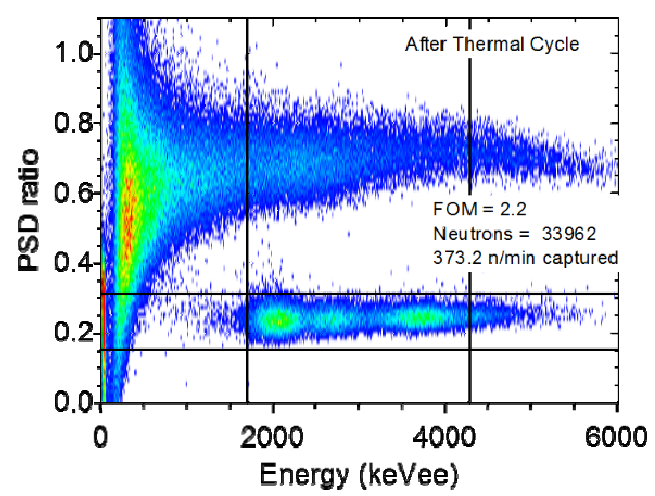

Figure 11. Plot of PSD ratio as a function of an approximate of the energy deposited in terms of the light yield from electrons.
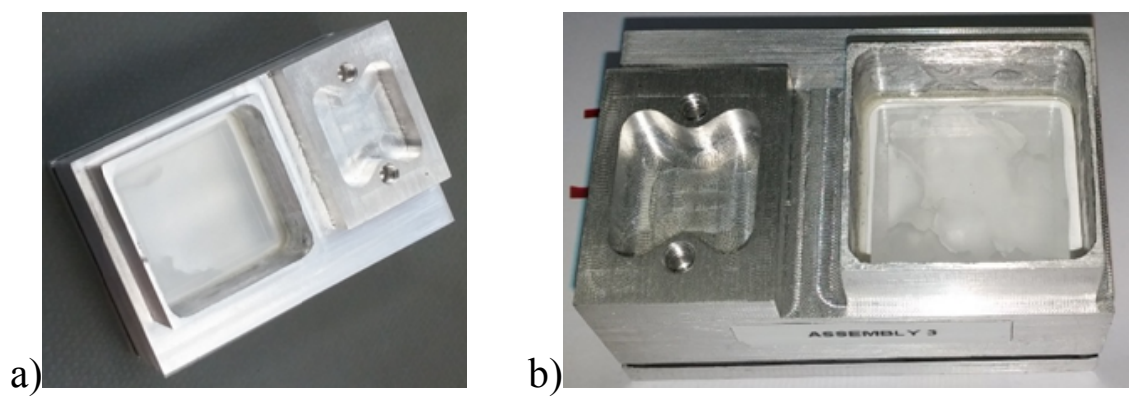

Figure 12. a) Picture of a prototype module with two stress fractures beneath the interface window. b) After temperature cycled, additional factures occur as shown in the picture.

Research into this issue resulted in a new module design to reduce the stress on the crystal. The fractures were generated when the changes in the sizes of the materials between the aluminum and the CLYC. The coefficient of expansion for $\mathrm{CLYC}$ is $34 \times 10^{-6}$ per ${ }^{\circ} \mathrm{C}$, while aluminum is $24 \times 10^{-6}$ per ${ }^{\circ} \mathrm{C}$. If the cyrstal is compressed into the package at a high 
pressure at room temperature, when the temperature is elevated, the additional strain will cause the crystal to fracture. The new packaging is shown in Figure 13. The package allows for the crystal to expand and contract into the softer packing materials used, while providing sufficient padding to prevent damage during launch. The PSD ratio plot is shown before and after thermal cycling. The Nevts Total (total neutron and gamma ray counts above $1.5 \mathrm{MeV}$ to 10 $\mathrm{MeV}$ ) and Counts within $3 \sigma$ (neutrons) can provide a measure on the degradation of the crystal. The ratio of Counts to Nevts provides a relative effeciency of the module to neutrons generated in the AmBe source. Gamma rays below 1.5 $\mathrm{MeV}$ are either from ${ }^{22} \mathrm{Na}, \mathrm{AmBe}$, or background. Above $1.5 \mathrm{MeV}$, the gamma rays are dominated by those from the AmBe source to get an measure of the AmBe activity. The ratio before the cycling is $0.627 \pm 0.007$ (stat) and after it is $0.626 \pm 0.007$ (stat).

a)

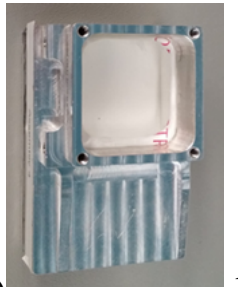

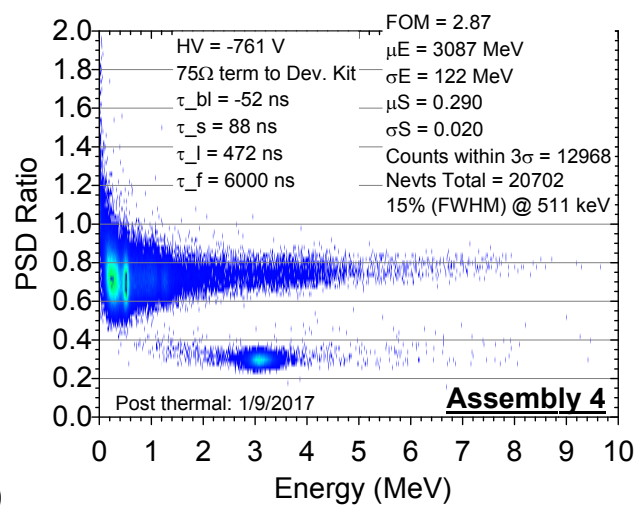

c)

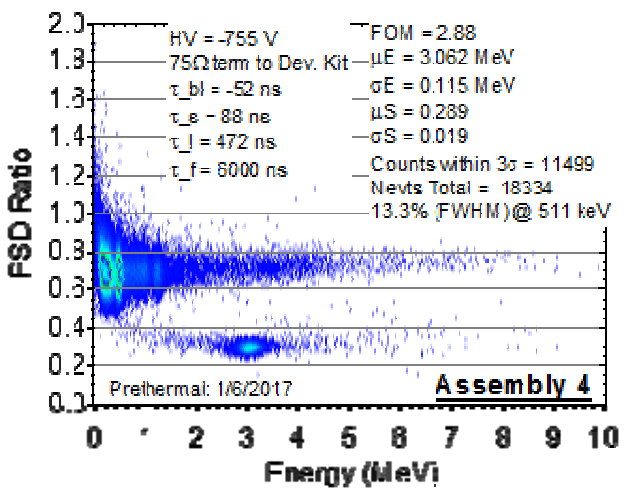

b)

PSD versus energy deposited prior to a thermal cycle. AmBe and ${ }^{22} \mathrm{Na}$ is used to generate the data. c) The plot of the PSD ratio versus energy deposited after thermal cycling.

\section{SUMMARY}

Research on the design and development of the LunaH-Map Mini-NS detector illustrates that a robust detector module that is ready for integration into the cubesat for spaceflight. The Mini-NS consists of various readout electronics to capture, process, and store data, as well as to communicate to the spacecraft for operation. These electronics are connected to analog electronics for signal amplification and bias control on photomultiplier tubes (PMT), where the analog electronics are thermally isolated from the higher power readout-processing electronics. The purpose to isolate the readout thermally is to reduce loading heat into the detector modules. Maintaining the heat below $10{ }^{\circ} \mathrm{C}$, the response of the detector will be sufficiently low to minimize systematic errors in counting neutrons. The analog electronics interface with the PMTs to readout the light pulses from the CLYC scintillators. These PMTs have been tested against vibration and are powered though a custom voltage divider network designed to reduce power consumption. The CLYC scintillators have undergone various thermal and vibrational testing, and these results indicate a high confidence that the crystals with the supporting packaging will survive the flight. These detector modules are covered with a cadmium sheet on the face pointing at the Moon and boron carbide on the other surfaces. The boron carbide is used to reduce all neutron counts that are backscattered to the detector, as these are considered background, and the cadmium will stop thermal neutrons, particularly those below $0.4 \mathrm{eV}$. Below this energy, water in the soil will enhance the thermal neutron count, but above this energy, water will suppress the neutron count. The Mini-NS is designed to measure the epithermal neutron counts with CLYC sensors.

\section{ACKNOWLEDGEMENTS}

We would like to thank the support from NASA for funding this project and for providing the SLS EM-1 launch vehicle to conduct this science. Though not listed as an author, a number of people on the LunaH Map Team has provided valued information on the design of the Mini-NS. Dr. Amanda Cook and Dr. Tony Colaprete at AMES provided support for our vibrational testing, which is much appreciated. We would also like to thank Ethan Cascio at MGH to help setup and run tests with the proton therapy beam. 


\section{REFERENCES}

[1] C. M. Pieters, J. N. Goswami, R. N. Clark, et al., "Character and Spatial Distribution of $\mathrm{OH} / \mathrm{H}<\mathrm{sub}>2</$ sub $>\mathrm{O}$ on the Surface of the Moon Seen by $\mathrm{M}<\sup >3</$ sup $>$ on Chandrayaan-1," Science, 326(5952), 568-572 (2009)

[2] R. N. Clark, "Detection of Adsorbed Water and Hydroxyl on the Moon," Science, 326(5952), 562-564 (2009)

[3] J. M. Sunshine, T. L. Farnham, L. M. Feaga, et al., "Temporal and Spatial Variability of Lunar Hydration As Observed by the Deep Impact Spacecraft," Science, 326(5952), 565-568 (2009)

[4] P. H. Schultz, B. Hermalyn, A. Colaprete, et al., "The LCROSS Cratering Experiment," Science, 330(6003), 468-472 (2010)

[5] A. Colaprete, P. Schultz, J. Heldmann, et al., "Detection of Water in the LCROSS Ejecta Plume," Science, 330(6003), 463-468 (2010)

[6] S. Maurice, D. J. Lawrence, W. C. Feldman, et al., "Reduction of neutron data from Lunar Prospector," Journal of Geophysical Research: Planets, 109(E7), n/a-n/a (2004)

[7] R. C. Elphic, V. R. Eke, L. F. A. Teodoro, et al., "Models of the distribution and abundance of hydrogen at the lunar south pole," Geophysical Research Letters, 34(13), n/a-n/a (2007)

[8] V. R. Eke, L. F. A. Teodoro, and R. C. Elphic, "The spatial distribution of polar hydrogen deposits on the Moon," Icarus, 200(1), 12-18 (2009)

[9] R. S. Miller, G. Nerurkar, and D. J. Lawrence, "Enhanced hydrogen at the lunar poles: New insights from the detection of epithermal and fast neutron signatures," Journal of Geophysical Research: Planets, 117(E11), n/a$\mathrm{n} / \mathrm{a}(2012)$

[10] I. Mitrofanov, M. Litvak, A. Sanin, et al., "Testing polar spots of water-rich permafrost on the Moon: LEND observations onboard LRO," Journal of Geophysical Research: Planets, 117(E12), (2012)

[11] E. B. Johnson, C. Whitney, S. Vogel, et al., "High Event Rate Studies on a CLYC-Based Neutron Detector Prototype," in INMM Annual Meeting, Indian Wells, CA, 2015. 